\title{
Zur Frage nach der Identität der milchkoagulierenden und proteolytischen Fermente.
}

\author{
Von \\ W. W. Sawitsch.
}

(Aus dem physiol. Laboratorium des Kais. Instituts für experimentale Medizin zu St. Petersburg.)

(Der Redaktion zugegangen am 3. Februar 1908.)

Ich Jahre 1904 erschien eine Arbeit von J. P. Pawlow und Parastschuk, (1) in der die Idee einer Identität der milchkoagulierenden Fermente mit den proteolytischen zum Ausdruck gebracht wurde. Zur Verteidigung ihres Standpunktes haben die Verfasser zahlreiche Beispiele für die Parallelität beider Wirkungen unter verschiedenen Bedingungen angeführt; in einigen Fällen ist es ihnen gelungen, eine durchaus genaue Proportionalität beider Funktionen nachzuweisen. Ferner wurde der eigenartige Einfluß der Alkalien auf das Pepsin beobachtet, welcher leicht eine Scheidung des Chymosins vom Pepsin vortäuschen konnte. Verschiedene Verfahren, die man vorgeschlagen hat, um das Chymosin vom Pepsin zu scheiden, wurden einer Kritik unterzogen (das Glässersche Verfahren, d. h. Fällung mittels essigsaurem Uranil; das Hammarstensche Verfahren, d. h. Fällung mittels $\mathrm{MgCO}_{3}$, sowie auch die Verdauung in einem sauren Milieu) und man erklärte die Ursachen, die die eine Wirkung verdeckten und die andere nicht beeinflußten. Schließlich zeigten die Verfasser, daß die Abhängigkeitsregeln der Wirkungszeit von der Menge des Fermentes sich je nach den Reaktionsbedingungen ändern. Auf diese Weise wird noch ein Einwand hinfällig, nämlich der, daß der Unterschied zwischen den Wirkungsgesetzen die Existenz zweier Fermentgruppen voraussetzt. Was diesen Punkt anbetrifft, so muß bemerkt werden, daß auch Sawjalow (2) zu denselben 
Schlußfolgerungen gelangt ist. Noch früher untersuchte Ganike ( $\left.{ }^{8}\right)$ die Wirkungsgesetze der proteolytischen Fermente in sehr verdünnten Lösungen mit Hilfe der Methoden der Zähigkeitsveränderung und fand, daß die Wirkung sich direkt proportionell zur Konzentration des Ferments verhält.

Auf diese Weise wurden zur weiteren Untersuchung zwei Wege angegeben: erstens muß der Einfluß der Alkalien auf das Pepsin genau studiert werden und zweitens, wenn die Koagulation und Proteolysis von einem und demselbén Agens abbängen, so muß zwischen diesen Wirkungen nicht nur eine grobe Parallelität, sondern eine genaue Proportionalität bestehen. Als die nächste Aufgabe erschien auf diese Weise die Erklärung der Proportionalität der beiden Wirkungen. Der Einfluß der Alkalien auf das Pepsin bildete den Gegenstand einer Arbeit Tichomirows. $\left({ }^{4}\right)$ Was die zweite Frage betrifft, so wurde uns dieselbe von Professor Pawlow vorgelegt.

\section{Kapit el. ${ }^{1}$ )}

Zur Bestimmung der Verdauungskraft wurden Eiweißröhrchen nach Mett oder Serumröhrchen benutzt; zur Koagulation verwendete man je $10 \mathrm{ccm}$ oder $5 \mathrm{ccm}$ frische Milch, die eine bestimmte Zeit im Wasserthermostaten gestanden hatte; die Gefäße wurden ausgekocht.

Bei der Untersuchung der Genauigkeit der Mettschen Methode für den Magensaft überzeugten wị uns, daß die Verdauung bei einem geringen Säuregehalt sich in günstigeren Bedingungen befindet, sich aber dem Schütz-Borissowschen Gesetz wenig unterordnet. Bei größeren Auflösungen mit Wasser erhielten wir einfache, aber keine quadratischen Verhältnisse. Für uns erwiesen sich die mittleren Säuregehalte von ungefähr $0,4 \% \mathrm{HCl}$ als geeigneter, für diese paßt das Schütz-Borissowsche Gesetz am besten. Nichtsdestoweniger hat das Mettsche Verfahren für die genaue Vergleichung der Pepsinmengen gewisse Mängel. Die Sache ist die, daß beim $\mathrm{Ab}$ zählen der Millimeter des verdauten Eiweißröhrchens immer

i) Der Inhalt dieses Kapitels bildete den Gegenstand eines Vortrags in der Gesellschaft russischer Ärzte im Oktober 1904. 
ein Irrtum bis $\mathrm{zu} 0,2 \mathrm{~mm}$ vorkommen kann. Ins Quadrat übertragen verdunkelt dieser Irrtum ganz bedeutend die wirkliche Menge des Ferments und es entsteht auf diese Weise gewissermaßen ein Auseinandergehen der koagulierenden und proteolytischen Wirkung. Wir beschlossen daher folgendermaßen vorzugehen:

Nachdem wir die Menge des Fermentes nach der Koagulation festgestellt haben, machen wir aus unseren Säften Mischungen, und zwar so, daß in einem bestimmten Volumen der Lösung eine gleiche Menge von Ferment und Säure enthalten ist. Es ist klar, daß solche äquivalente Mischungen die Milch in der gleichen Zeit zum Gerinnen bringen müssen; zum Verdauen gebracht, müssen sie die gleiche Menge Millimeter von den Eiweiß- oder Serumröhrchen verdauen. Wir stellten also solche Mischungen her und ließen durch sie gerinnen und verdauen, wobei wir sie im letzten Falle manchmal gleichmäßig mit Säure verdünnten.

Zuerst untersuchten wir verschiedene stündliche Portionen der Säfte, die nach Verabreichung von Brot, Fleisch, Milch genommen wurden. Die Säfte wurden nach Stunden gesammelt, dann wurden sie durch Verdünnung auf einen Säuregehalt von $4 \%$ gebracht und dann je nach dem Fermentgehalt aufgelöst.

Aus der Tabelle I ersehen wir ein vollständiges Zusammenfallen der beiden Wirkungen in den äquivalenten Mischungen. Die Verhältnisse der Menge an Fermenten, die auf dem Wege der Koagulation erhalten wurden, kommen den Quadratverhältnissen der Millimeter verdauter Eiweißröhrchen sehr nahe. Es muß jedoch bemerkt werden, daß man nur nach den Speisesorten vergleichen darf, weil in der Tabelle Versuche aufgenommen wurden, die man an verschiedenen Tagen und mit verschiedenen Milchsorten angestellt hatte.

\section{Tabelle I.}

Die auf Brot genommenen Saftportionen sind $16 \mathrm{mal}$ verdünnt und diese verdünnten Portionen der ersten Stunde sind durch $A_{1}$, die der zweiten durch $A_{2}$ usw. bezeichnet. Die stündlichen auf Fleisch genommenen Saftportionen sind $8 \mathrm{mal}$ verdünnt und durch $B_{1}, B_{2}$ usw. bezeichnet. Die auf Milch genommenen Säfte sind 4 mal verdünnt und 
Über milchkoagulierende und proteolytische Fermente.

durch $\mathrm{C}_{\mathfrak{t}}, \mathrm{C}_{\mathrm{q}}$ usw. bezeichnet. Zur Koagulation nahm man je 1,0 der äquivalenten Mischung, außer bei der zweiten Bestimmung, der des Fleischsaftes, bei der man 0,7 nahm.

\begin{tabular}{|c|c|c|c|c|c|c|c|c|c|c|c|}
\hline $\begin{array}{c}\text { Por- } \\
\text { tion } \\
\text { des } \\
\text { Saftes }\end{array}$ & \multicolumn{2}{|c|}{$\begin{array}{c}\text { Verdaute } \\
\text { mm } \\
\text { Eiweiß- } \\
\text { röhrchen }\end{array}$} & $\begin{array}{c}\text { Quadrat- } \\
\text { verhältnis } \\
\text { nach der } \\
\text { Ver- } \\
\text { dauung }\end{array}$ & $\begin{array}{l}\text { Relative } \\
\text { Ferment- } \\
\text { menge } \\
\text { nach der } \\
\text { Koagula- } \\
\text { lation }\end{array}$ & \multicolumn{3}{|c|}{$\begin{array}{l}\text { Zusammensetzung } \\
\text { der äquivalenten } \\
\text { Mischungen }\end{array}$} & \multicolumn{2}{|c|}{$\begin{array}{l}\text { Die } \\
\text { Äquivalente } \\
\text { machten } \\
\text { gerinnen } \\
\text { I | II }\end{array}$} & \multicolumn{2}{|c|}{$\begin{array}{l}\text { Die Mi- } \\
\text { schungen } \\
\text { verdauten }\end{array}$} \\
\hline$A_{1}$ & $3,8 \mathrm{~m}$ & & 5,0 & 4,8 & 0,21 & Saft $+0,79 \mathrm{~S}$ & äure & $4^{\prime} 20^{\prime \prime}$ & $4^{\prime} 30^{\prime \prime}$ & 2,0 & $\mathrm{~nm}$ \\
\hline$A_{2}$ & 3,2 & $\triangleright$ & 3,5 & 3,3 & 0,3 & $>+0,7$ & & $4^{\prime} 10^{\prime \prime}$ & $4^{\prime} 25^{\prime \prime}$ & 2,0 & , \\
\hline$A_{8}$ & 2,7 & > & 2,5 & 2,3 & 0,44 & $>+0,56$ & & $4^{\prime} 20^{\prime \prime}$ & $4^{\prime} 20^{\prime \prime}$ & 1,9 & , \\
\hline $\mathbf{A}_{4}$ & 2,8 & $>$ & 2,7 & 2,6 & 0,39 & $>+0,61$ & & $4^{\prime} 25^{\prime \prime}$ & $4^{\prime} 50^{\prime \prime}$ & 1,9 & , \\
\hline$A_{8}$ & 1,7 & > & 1,0 & 1,0 & 1,0 & 一 & & $4^{\prime} 10^{\prime \prime}$ & $4^{\prime} 15^{\prime \prime}$ & 1,9 & , \\
\hline$B_{1}$ & 5,7 & > & 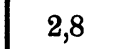 & 2,5 & 0,4 & Saft $+0,6 \quad S$ & äure & 一 & $250^{\prime \prime}$ & 2,8 & \\
\hline$B_{2}$ & 2,7 & $\triangleright$ & 1,5 & 1,5 & 0,65 & $\Rightarrow+0,3 \grave{~}$ & & $115^{\prime \prime}$ & $230^{\prime \prime}$ & 2,6 & , \\
\hline $\mathrm{B}_{\mathbf{8}}$ & 2,2 & > & 1,0 & 1 & 10 & - & & $105^{\prime \prime}$ & $235^{\prime \prime}$ & 2,7 & , \\
\hline $\mathrm{B}_{4}$ & 2,6 & > & 1,4 & 1,4 & 0,71 & $>+0,29$ & 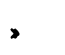 & $115^{\prime \prime}$ & $215^{\prime \prime}$ & 2,7 & , \\
\hline $\mathbf{B}_{6}$ & 3,3 & $>$ & 2,25 & 2,3 & 0,43 & $>+0,57$ & 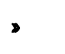 & $105^{\prime \prime}$ & $240^{\prime \prime}$ & 2,7 & , \\
\hline $\mathrm{C}_{1}$ & 2,3 & , & 2,7 & 2,8 & 0,36 & Saft $+0,64 \mathrm{~S}$ & oute & $180^{\prime \prime}$ & $175^{\prime \prime}$ & 2,0 & \\
\hline $\mathrm{C}_{2}$ & 1,4 & , & 1,0 & 1,0 & 1,0 & - & & $160^{\prime \prime}$ & $165^{\prime \prime}$ & 2,1 & , \\
\hline $\mathrm{C}_{3}$ & 2,1 & , & 2,2 & 2,3 & 0,43 & $=+0,57$ & , & $175^{\prime \prime}$ & $165^{\prime \prime}$ & 2,0 & 3 \\
\hline $\mathrm{C}_{4}$ & 3,2 & , & 5,2 & 5,0 & 0,2 & $\therefore+0,8$ & $\triangleright$ & $160^{\prime \prime}$ & $180^{\prime \prime}$ & 2,1 & , \\
\hline $\mathrm{C}_{5}$ & 3,0 & , & 4,6 & 4,3 & 0,23 & $=+0,77$ & , & $175^{\prime \prime}$ & $175^{\prime \prime}$ & 2,0 & $>$ \\
\hline
\end{tabular}

Wir haben die von verschiedenen Speisen genommenen Säfte nicht untereinander verglichen, weil dies schon in der Arbeit von Pawlow und Parastschuk geschehen ist. Wir haben uns deshalb darauf beschränkt, die stärkste vom Brote erhaltene Portion mit der schwächsten von Milch erhaltenen Portion zu vergleichen. Wir stellten Äquivalentmengen her: 0,54 Brotsaft, 32 mal verdünnt $+0,46$ Säure (A) und 1,0 Milchsaft, 2 mal verdünnt (B). A koagulierte in $2^{\prime} 35^{\prime \prime}$ und verdaute 2 . 1 Eiweißröhrchen; B koagulierte Milch in $2^{\prime} 35^{\prime \prime}$ und verdaute 2,0; auf diese Weise ist der Milchsaft 29,6 mal schwächer als der Brotsaft, sowohl in bezug auf die Koagulation, als auch auf die Proteolysis. 
Gegen die erhaltenen Resultate ließe sich einwenden, daß das Zusammenfallen der beiden Funktionen nicht von der Identität des Chymosins mit dem Pepsin abhängt, sondern von der Parallelität der Absonderung beider Fermente. Wir verfolgten daher beide Wirkungen unter verschiedenen künstlichen Bedingungen. Wir erwärmten zuerst die sauren Lösungen bis zu verschiedenen Temperaturen und untersuchten dann beide Funktionen; zweitens stellten wir einen Teil des Saftes in den Thermostaten, der andere Teil, der zur Kontrolle diente, wurde kalt gestellt; beide Wirkungen wurden dann verglichen. Wären es nun zwei verschiedene Fermente, so müßte eine Wirkung verloren gehen und die andere erhalten bleiben, und dies um so mehr, da das Stehen der sauren Pepsinlösung im Thermostaten als ein Mittel angeführt wurde, um das Pepsin vom Chymosin zu trennen.

\section{Tabelle II.}

Es wurde Magensaft nach der Scheinfütterung genommen, bis zu einem Säuregehalt von $4 \%$ verdünnt; Teil I wurde in der Kälte stehen gelassen, der übrige wurde im Wasserbade erwärmt, und zwar Portion II bis auf $53^{\circ}$, III bis auf $55^{\circ}$, IV bis auf $56^{\circ}, \mathrm{V}$ bis auf $57^{\circ}$ und VI bis auf $58^{\circ}$. Wegen der geringen Fermentmenge in Portion VI verglich man zuerst die ersten 5 Portionen und dann am anderen Tage I, V und VI.

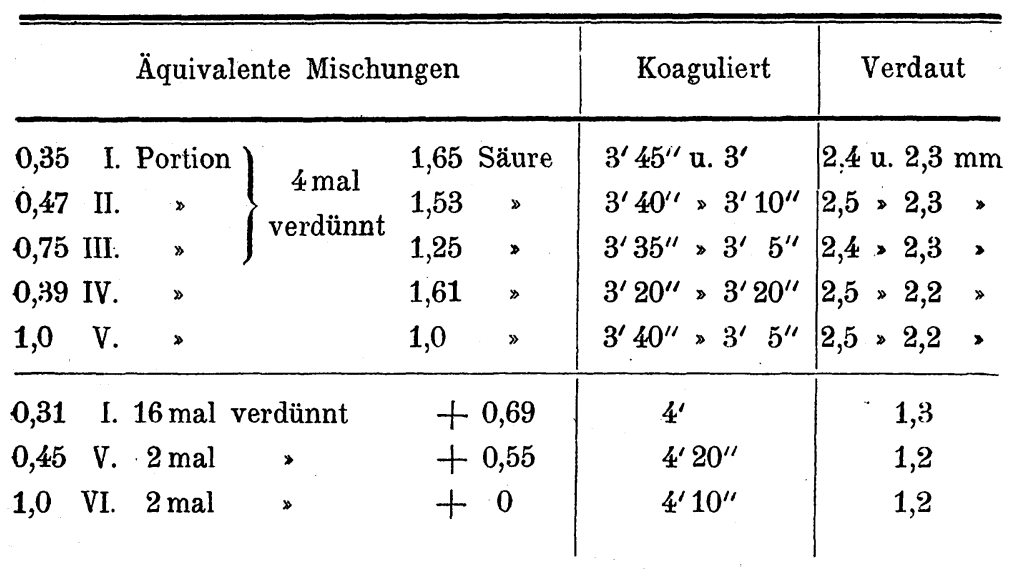

Also geht bei der Erwärmung des sauren Magensaftes bis $z u$ verschiedenen Temperaturen die Verminderung beider Funk- 
tionen vollständig gleichmäßig vor sich. Auch aus dem Stehen im Thermostaten bei $38^{\circ}$ ergab sich das gleiche Resultat, was aus dem unten angeführten Versuch ersichtlich ist. Wir nahmen Magensaft, verdünnten ihn bis zu $4 \%$ Säuregehalt, ein Teil wurde ị die Kälte gestellt (K), den anderen (T) stellte man in den Thermostaten. Nach 3, 5, 8 und 11 Tagen wurden. Proben genommen und mit dem kalt gestellten auf 2 Arten verglichen; erstens wurde der saure Saft durch 2 Volumen Säure verdünnt und zur Verdauung gebracht und zwar immer auf 10 Stunden; zweitens wurden die Portionen $\mathrm{K}$ und $\mathrm{T}$ vorsichtig und unter fortwährendem Umrühren mit $\mathrm{HNaCO}_{3}$ in substantia neutralisiert und ungefähr 2 Stunden bei häufigem Umrühren zwecks Absonderung von $\mathrm{CO}_{2}$ stehen gelassen und dann mit neutralen Lösungen Äquivalente hergestellt, welche nach Verdünnung mit 2 oder 4 Volumen Säure auf verschiedene Zeit zur Verdauung gestellt wurden. Zur Koagulation nahm man je 10,0 Milch, erst nach 11 Tagen je 5,0; weiter wurde der Versuch nicht fortgesetzt, da sich die Koagulation sehr verzögert hatte und es schwer fiel, ohne Mittel, die das Gerinnen be-

Tabelle III.

\begin{tabular}{|c|c|c|c|c|c|}
\hline $\begin{array}{l}\text { Stehzeit } \\
\text { im Ther- } \\
\text { mostaten }\end{array}$ & \begin{tabular}{|} 
Verdau- \\
ung der \\
sauren \\
Säfte nach \\
10 Stund.
\end{tabular} & $\begin{array}{c}\text { Quadrat- } \\
\text { ver- } \\
\text { hältnis }\end{array}$ & $\begin{array}{c}\text { Wieviel mal } \\
\mathbf{K} \text { verdünnt } \\
\text { wurde, um das } \\
\text { Äquivalent } \\
\text { zu erhalten }\end{array}$ & $\begin{array}{c}\text { Zeit der } \\
\text { Koagulation } \\
\text { der } \\
\text { äquivalenten } \\
\text { Mischung }\end{array}$ & $\begin{array}{l}\text { Verdauung } \\
\text { der Äqui- } \\
\text { valente nach } \\
\text { der Ver- } \\
\text { dünnung mit } \\
\text { Säuren }\end{array}$ \\
\hline $\begin{array}{c}3 \\
\text { Tage }\end{array}$ & $\begin{array}{l}\mathrm{K} 2,9 \mathrm{~mm} \\
\mathrm{t} 2,4\end{array}$ & 1,4 & 1,5 & $\begin{array}{ll}\mathrm{K}^{\prime} & 4^{\prime} \\
\mathrm{t} & 3^{\prime} 50^{\prime \prime}\end{array}$ & $\begin{array}{ll}\mathrm{K} & 1,8 \mathrm{~mm} \\
\mathrm{t} & 1,7\end{array}$ \\
\hline $\begin{array}{c}5 \\
\text { Tage }\end{array}$ & $\begin{array}{l}\mathrm{K} 2,6 \\
\mathrm{t} 1,8\end{array}$ & 2,0 & 1,8 & $\begin{array}{ll}\mathrm{K} & 3^{\prime} 50^{\prime \prime} \\
\mathrm{t} & 4^{\prime}\end{array}$ & $\begin{array}{ll}\mathrm{K} & 1,9 \\
\mathrm{t} & 1,7\end{array}$ \\
\hline $\begin{array}{c}8 \\
\text { Tage }\end{array}$ & $\begin{array}{l}\mathrm{K} 2,9 \\
\mathrm{t} \quad 1,3\end{array}$ & 4,9 & 4,25 & $\begin{array}{l}\text { K } 12^{\prime} 40^{\prime \prime} \\
\text { t } 1230^{\prime \prime}\end{array}$ & $\begin{array}{ll}\mathrm{K} & 1,6 \\
\mathrm{t} & 1,7\end{array}$ \\
\hline $\begin{array}{c}11 \\
\text { Tage }\end{array}$ & $\begin{array}{l}\mathrm{K} 2,8 \text { " } \\
\mathrm{t} 0,85\end{array}$ & 10,9 & 11,7 & $\begin{array}{ll}K & 23^{1 / z^{\prime}} \\
t & 22^{1} / z^{\prime}\end{array}$ & $\begin{array}{ll}\mathrm{K} & 0,9 \\
\mathrm{t} & 1,0\end{array}$ \\
\hline
\end{tabular}


schleunigen, auszukommen, deren Anwendung wir vermeiden wollten. Wir sahen, daß sich bei beiden Verfahren ähnliche Verhältnisse ergaben; die größte Abweichung ergab 4,9 nach der Verdauung und 4,25 nach der Koagulation, doch liegt auch hier der Fehler in der beschränkten Genauigkeit des Mettschen Verfahrens, denn nimmt man 1,4 statt 1,3 , so erhält man eine vollkommene Übereinstimmung $(4,3)$; folglich beträgt der Fehler 0,1.

Hieraus wird klar, daß beim Stehen der sauren Magensäfte im Thermostaten eine progressive, vollkommen gleichmäßige Verminderung beider Wirkungen stattfindet.

Andere Autoren erhielten ein ganz anderes Resultat. Zum Teil hing dies davon ab, daß man bei der Koagulation und Proteolysis Proben von verschiedener Empfindlichkeit benutzte; infolgedessen fand gewissermaßen eine Differenz statt. Andererseits hing es von der unsorgfältigen Einrichtung der Versuche ab. So erhielt man z. B. bei Hemmeter $\left(^{5}\right)$ beim Stehen im Thermostaten (Fall Werner) von 1 II bis 26 II sogar ein Anwachsen der Verdauungskraft von 3,0 bis 3,5, während die Koagulation sich von $5^{\prime}$ auf $15^{\prime}$ verlangsamte. Dies erklärt sich leicht durch die ungenügende Verdünnung des Mageninhaltes und die daraus folgende unrichtige Berechnung der Pepsinmenge, worauf, besonders für den Mageninhalt, Nierenstein und Schiff $\left({ }^{6}\right)$ hingewiesen haben.

Bei Hemmeter verminderte sich dann der freie Säuregehalt; so war (Fall Spencer) zu Anfang des Versuches 60, gegën das Ende aber 47 vorhanden, während der allgemeine Säuregehalt von 64 auf 74 anwuchs. Es war mit anderen Worten eine ganze Reihe von Ungenauigkeiten vorhanden.

Schon in der Arbeit Pawlows und Parastschuks ist darauf hingewiesen worden, daß die Verminderung beider Funktionen des aktivierten Pankreassaftes durch das Stehen im Thermostaten ganz gleichmäßig vor sich geht; jetzt war es nun interessant, die relative Schnelligkeit der Zerstörung aller Fermente des Saftes kennen zu lernen. Wir nahmen zu dem Versuche den Pankreassaft von einem akuten Versuche, fügten 10\% Darmsaft hinzu; die eine Portion wurde sofort in den 
Schnee gestellt (A), die andere wurde bei $38^{\circ}$ bis $20^{\prime}$ (B) stehen gelassen und die dritte 3 Stunden (C). Das Fettferment wurde durch Titration der Fettsäure bestimmt, die sich durch Scheidung von 10,0 1\% iger Monobutyrinlösung und 0,3 Saft gebildet hatte. Die Amylase bestimmte man durch Stärkeröhrchen nach Walter, wobei man 0,1 Saft auf 1,0 Soda zu $0,3 \%$ nahm, die Portionen A und B wurden vorher $10 \mathrm{mal}$ mit physiologischer Kochsalzlösung verdünnt. Zur Koagulation nahm man 5,0 Milch, die mit 0,5 Salzsäure $(0,5 \%)$ angesäuert war; Saft nahm man 0,2. Zur Verdauung wurde die äquivalente Mischung zweimal mit $0,3 \%$ Soda verdünnt; die Verdauung fand bei Zimmertemperatur statt.

Tabelle IV.

\begin{tabular}{|c|c|c|c|c|c|c|}
\hline & $\begin{array}{l}\text { Titr. Menge } \\
\text { für die } \\
\text { Neutrali- } \\
\text { sation }\end{array}$ & $\begin{array}{c}\text { Ver- } \\
\text { dauung } \\
\text { der } \\
\text { Stärke- } \\
\text { röhrchen }\end{array}$ & $\begin{array}{l}\text { Verdünnung } \\
\text { zur Herstel- } \\
\text { lung der } \\
\text { Äquivalente }\end{array}$ & $\begin{array}{c}\text { Zeit der } \\
\text { Koagu- } \\
\text { lation der } \\
\text { Äqui- } \\
\text { valente }\end{array}$ & $\begin{array}{r}\text { Verda } \\
\mathrm{d} \\
\text { Misch } \\
\text { Serum }\end{array}$ & $\begin{array}{l}\text { auung } \\
\text { er } \\
\text { ungen } \\
\text { Eiweiß }\end{array}$ \\
\hline A & 1,1 & $1,9 \mathrm{~mm}$ & 3,6 & $3^{\prime} 15^{\prime \prime}$ & $5,2 \mathrm{~mm}$ & $1,0 \mathrm{~mm}$ \\
\hline B & 0,5 & 1,7 , & 3,1 & $3^{\prime} 10^{\prime \prime}$ & 5,0 , & 1,1, \\
\hline $\mathrm{C}$ & 0,3 & 1,4 & 1 & $3^{\prime} 30^{\prime \prime}$ & 5,0 , & 1,0 \\
\hline $\begin{array}{c}\text { Reines } \\
\text { Monobut. }\end{array}$ & 0,1 & - & - & - & - & - \\
\hline
\end{tabular}

Der von uns gefundene Gang der Zerstörung der Fermente im Pankreassaft stimmt mit den nach einer anderen Methode von Ganike ( $\left.{ }^{7}\right)$ erhaltenen Resultaten überein. Die Zerstörung des Steapsins geht energisch während der ersten $20^{\prime}$ vor sich, später finden sich nur noch Spuren. Amylase bleibt während der ersten $20^{\prime}$ erhalten, dann tritt eine rasche Zerstörung ein. Trypsin wird langsamer zerstört, der Prozeß geht allmählich und beständig vor sich. Das Sinken der Verdauungskraft fällt mit der Verminderung der koagulierenden Kraft zusammen. Unsere Ergebnisse stimmen auch gut mit dem von Vernon(8) erhaltenen Resultat überein, daß bei konsequenter Fällung der Pankreasextrakte mit Alkohol die Trypsinwirkung mit der koagulierenden Wirkung parallel läuft; während die diastatische scharf abweicht. Außerdem konnten wir eine voll- 
kommene Proportionalität der milchkoagulierenden und proteolytischen Wirkung in Pankreassäften konstatieren, die unter verschiedenen Bedingungen genommen wurden, aber in Anbetracht dessen, daß sich die Fermente im Pankreassaft überhaupt parallel absondern, haben wir diesen Angaben keine Ziffern hinzugefügt.

\section{Kapitel.}

Wir gehen jetzt zu den natürlichen Säften über, die Pepsin in alkalischer Lösung enthalten, $d$. h. in Säften aus dem Pylorus und dem Brunnerschen Teile des Darmes. In diesem Teile der Arbeit bedienten wir uns der Angaben Tichomirows beziehentlich des Einflusses der Alkalien auf das Pepsin. Wir bewirkten das Erscheinen des Ferments durch Säure, die in einer solchen Menge hinzugegossèn wurde, daß sie ${ }^{4 / 5}$ der gesamten Alkalität zu neutralisieren imstande wạr, oder wir gossen die Säure vorsichtig über den Saft hin, wobei die Ansäurung langsam durch Diffusion vor sich ging; auch hiermit erzielten wir keine schlechten Resultate. 'Es muß bemerkt werden, daß die Säfte sehr dick sind, und zur Bestimmung der Verdauungsfähigkeit haben wir sie stark verdünnt. So wurde der Brunnersche Saft durch Diffusion angesäuert und dann auf 4\% Säuregehalt gebracht; auch der Magensaft wurde bis auf $4 \%$ verdünnt. Zur Koagulation verwandte man 10,0 Milch und 0,8 Saft. Man stellte äquivalente Mischungen her. Der Brunnersche Saft koagulierte in $3^{\prime} 40^{\prime \prime}$, der Magensaft in $3^{\prime} 45^{\prime \prime}$.

Tabelle V.

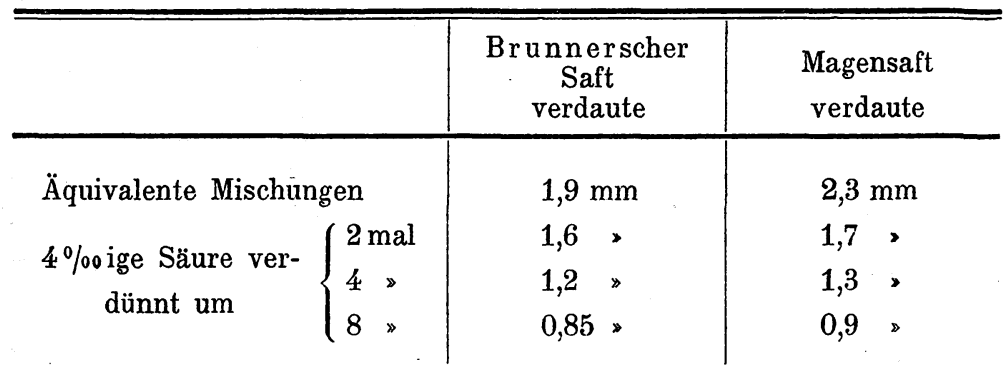

Wie aus der Tabelle ersichtlich ist, fand die Hemmung der Verdauung im Brunnerschen Safte infolge der Dichtigkeit 
dieses Saftes statt und verschwand mit der Verdünnung; es tritt eine vollkommene Proportionalität zwischen der koagulierenden und der auflösenden Funktion zutage. Beim pylorischen Saft erklärt sich die Sache anders. Nachdem wir ihn ebenso wie den Brunnerschen Saft angesäuert und nach der Koagulation mit Magensaft eine äquivalente Mischung hergestellt hatten, waren wir darüber erstaunt, daß der pylorische Saft im Vergleich mit der koagulierenden Kraft bedeutend stärker verdaute als der Magensaft.

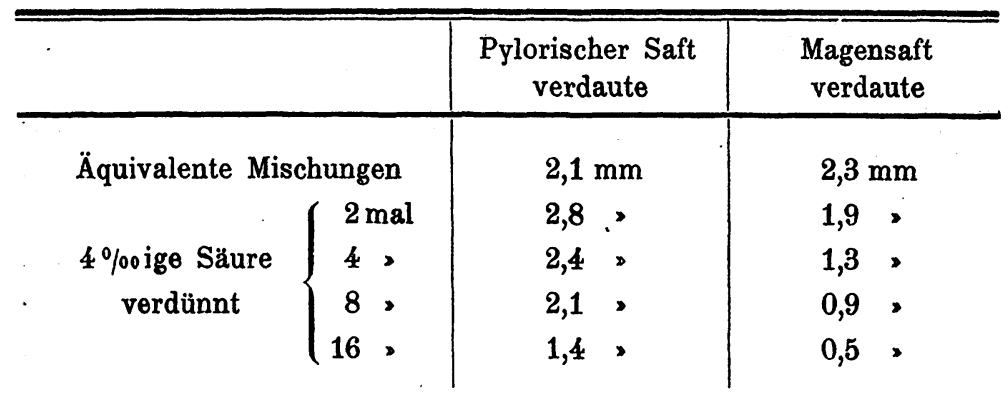

Hier lenkt jene Tatsache die Aufmerksamkeit auf sich, daß erst nach einer 8 fachen Verdünnung die richtigen Verhältnisse (nach Schütz-Borissow) beobachtet werden. Anderseits konnten wir am anderen Tage bei der Untersuchung der Äquivalente auf das Verdauen der Mischung hin bemerken, daß der pylorische schneller und manchmal sogar bedeutend schneller koaguliert als der Magensaft. Als wir die Ursache dieser Erscheinung untersuchten, richteten wir unsere Aufmerksamkeit darauf, daß der pylorische Saft homogen zu sein schien; wenn man ihn aber mit Indikator Lacmoid titrierte, so bemerkte man violett gefärbte Flocken zu einer Zeit, in der die Flüssigkeit noch rot ist. Bei der Verdünnung nehmen diese Flocken an Zahl ab. Wir nehmen an, daß hier ein Rätsel verborgen ist, daß nämlich das Ferment in diesen Klümpchen eingeschlossen ist, die sich dann bei der Verdünnung nach und nach auflösen. Deshalb verdünnten wir den pylorischen Saft so lange, bis das Schütz-Boriss owsche Gesetz seine Kraft nahm, und erst dann stellten wir die äquivalenten Mischungen her. So verdaute der 
pylorische Saft 1,7, viermal verdünnt 2,5 , achtmal verdünnt 1,8 . Hierauf stellten wir Äquvalente her nach der Koagulation des Magensaftes mit achtmal verdünntem pylorischem Saft. Der pylorische Saft koagulierte in $7^{\prime} 10^{\prime \prime}$, der Magensaft in $7^{\prime}$; der erstere verdaute 1,0, der letztere 0,8. Ferner begannen wir zu dem alkalischen pylorischen Saft eine Sodalösung hinzuzufügen, um eine bessere Auflösung der schleimigen Massen zu erzielen; dann fügten wir nach Tichomirow Säure hinzu und manchmal wurde auch zur Entfernung des Überschusses an Salzen dialysiert. Auch in diesem Falle trat ein vollständiges Zusammenfallen beider Funktionen ein.

1,0 Magensaftäquivalent koagulierte in $6^{\prime}$, verdaute $2,5 \mathrm{~mm}$ Eiweißröhrchen; das Äquivalent des pylorischen Saftes koagulierte in $6^{\prime} 20^{\prime \prime}$, verdaute $2,6 \mathrm{~mm}$, viermal verdünnt $-1,2 \mathrm{~mm}$.

Als der pylorische Saft der Fäulnis unterworfen wurde, erreichten wir ebenfalls eine Verminderung der Dichtigkeit und das Verschwinden der oben erwähnten Klümpchen. Man gelangt dabei leicht $z u$ einer Proportionalität beider Funktionen.

Auf diese Weise können wir das Auseinandergehen der beiden Funktionen dadurch erklären, daß die Schleimklümpchen das Ferment in sich einschließen, welches nur allmählich in einem sauren Milieu in Lösung übergeht; in der Milch findet dies nicht statt oder es geht sehr langsam. Die Klümpchen verdecken gewissermaßen das Ferment.

Wir machen darauf aufmerksam, daß unseren Versuchen gemäß der pylorische Saft bedeutend reicher an Ferment ist, als früher angenommen wurde. Die Verdauungskraft des pylorischen Saftes wurde von den Autoren mindestens um fünfmal geringer geschätzt, als sie in Wirklichkeit ist.

\section{Kapitel.}

Wir sahen also bei den natürlichen Säften eine vollkommene Proportionalität der beiden Wirkungen. Verhalten sich nun die Präparate der Fermente ebenso? Wir untersuchten käufliche Pepsinpräparate (pepsinum hydrochloratum solubile 100,0\% Merk und pepsinum gallicum acidifié Bondault). Nachdem wir nach der Koagulation äquivalente Mischungen 
aus Pepsinpräparaten und aus Magensaft hergestellt hatten, sahen wir beständig, daß die Proteolysis in den Magensäften bedeutend stärker war (ungefähr 11/2 mal so stark), sogar wenn wir die Antipepsinmenge dadurch ausgeglichen hatten, daß wir zu der einen Lösung die andere gekochte Lösung hinzufügten. Obgleich man ein scheinbares Auseinandergehen der Wirkungen erhielt, gelang es uns durch wiederholte Reinigung mit Spiritus dennoch, Präparate zu erhalten, bei denen ein vollständiges Zusammenfallen beider Funktionen stattfand. Als Äquivalente nahm man erstens das mit Spiritus gereinigte und mit Wasser verdünnte Präparat Merk; und zweitens Magensaft, der vorsichtig mit $\mathrm{NaHCO}_{3}$ neutralisiert und verdünnt war; 0,5 Magensaft koagulierte in $4^{\prime} 30^{\prime \prime} ; 0,5$ Präparat koagulierte in $4^{\prime} 20^{\prime \prime}$. Hierauf wurden die Säfte stark mit Säure verdünnt; der Magensaft verdaute 1,5 Eiweißröhrchen und 4,8 Serumstäbchen, das Präparat verdaute 1,3 der ersteren und 4,6 der letzteren.

Genau ebenso verfuhren wir mit dem anderen Pepsinpräparat, nur fügten wir in Anbetracht der geringen Fermentmenge auf 5,0 Milch $1,0 \mathrm{ccm} 5 \% \mathrm{CaCl}_{2}$ hinzu. Das Äquivalent des Präparates koagulierte in $7{ }^{\prime} 10^{\prime \prime}$ und nach der Verdünnung mit Säure verdaute es 2,2 Serumstäbchen. Das Äquivalent des Magensaftes koagulierte in 7' und verdaute 2,0. Ferner sammelten wir das Spiritusfiltrat, dämpften es aus, verdünnten es mit Wasser, kochten es, filtrierten es und probierten, wie es auf die Koagulation einwirkt. $\mathrm{Zu}$ einer Portion Milch fügten wir 0,5 Wasser hinzu, zu der anderen 0,5 der gewonnenen Lösung; hierauf fügten wir 0,2 Magensaft hinzu. Die erste Portion koagulierte in $3^{\prime} 45^{\prime \prime}$, die zweite in $2^{\prime} 15^{\prime \prime}$. Schließlich stellten wir 2 Mischungen zur Verdauung: 1,0 Magensaft $+1,0$ Wasser und 1,0 von demselben Magensaft $+1,0$ Lösung aus dem Spiritusfiltrat. Die erste Mischung verdaute $4,0 \mathrm{~mm}$, zu 0,7 genommen koagulierte sie in 50"; die zweite Mischung verdaute 3,5 und koagulierte in 30". Auf diese Weise erklärt sich das Auseinandergehen der beiden Funktionen durch das Vorhandensein von Stoffen, die die Verdauung verzögern, in noch größerem Maße aber durch das Vorhandensein von Substanzen, die die Koagulation beschleunigen. Überhaupt muß man darauf 
achten, daß sich in den Extrakten immer große Mengen derartiger Stoffe befinden, während in den reinen Säften ${ }^{1}$ ) sehr wenige vorhanden sind. Es ist daher sehr schwer, mit Extrakten $\mathrm{zu}$ arbeiten und sehr leicht, ein Auseinandergehen der beiden Funktionen $\mathrm{zu}$ erhalten. Außerdem ist uns noch ein interessanter Fall vorgekommen, der sehr leicht ein Auseinandergehen beider Funktionen hätte vortäuschen können. Wir hatten ein käufliches Präparat flüssigen Labferments bekommen, das vorzüglich koagulierte und schlecht verdaute. Die Ursache hiervon war zum Teil eine alkalische Reaktion und ferner das Vorhandensein von Antipepsinen und von Stoffen, die die Koagulation beschleunigen. Zieht man alles dies in Betracht, so erhielten wir statt der Spuren einer Verdauung eine ganz ordentliche Verdauung, aber eine geringere, als mit den Magensäften nach dem Äquivalent der Koagulation. Als Ursache hiervon ergab sich die schnelle Zerstörung des Fermentes durch das Stehen in saurer Lösung. So koagulierten $0,6 \mathrm{Lab}$, bis zu $2 \%$ angesäuert, 10,0 Milch in 2'35", nachdem sie 18 Stunden im Thermostaten gestanden batten - in $20^{\prime} 40^{\prime \prime}$, der Äquivalent aber des Magensaftes vor dem Stehen in Thermostaten in 2'25" nach dem Stehen - in $2^{\prime} 40^{\prime \prime}$. Auf diese Weise muß man einen besonderen, wenig widerstandsfähigen Zustand des Fermentes annehmen, den labilen Zustand. Einen analogen $\mathrm{Zu}-$ stand erhielten wir, wenn wir den dialysierten Magensaft mit Cholestearin nach Schrumpf $\left(^{9}\right)$ bearbeiteten. Dieser Autor bearbeitete den dialysierten Preßsaft mit Cholestearin und erhielt ein Pepsinpräparat ohne Eiweiß (negative biuretische Reaktion). Dieses Pepsin verdaute ganz gut, koagulierte aber nicht. Ferner bemerkte der Verfàsser auch eine leichte Zerstörung dieses Pepsins. Wir wiederholten diese Versuche, nur mit dem Unterschiede, daß wir dialysierten Magensaft nahmen und ein Pepsinpräparat erhielten, das sich ebenfalls beim Stehen schnell zerstörte. Dennoch konnten wir eine milchkoagulierende Wirkung entdecken, obwohl dieselbe allerdings erst nach 3-4 Stunden eintrat. Wenn wir aber zu der Milch Säure, oder noch besser

1) Unter dem Wort «Saft verstehen wir nur natürliche Sekrete, aber nicht Extrakte. 
$\mathrm{CaCl}_{2}$, hinzufügten, um die Wirkung im sauren Milieu zu vermeiden, so erhielten wir eine vorzügliche Koagulation nach $5^{\prime}$. Wenn wir nach der Kogulation äquivalente Mischungen aus Magensaft und Pepsin nach Schrumpf hergestellt hatten, so sahen wir immer, daß der Magensaft bedeutend mehr verdaute, während das gereinigte Labpräparat und das Pepsin Verdauungsresultate ergaben, die einander ähnlich waren. Die Ursache dieses labilen Zustandes des Ferments müssen wir in einer Abspaltung des Ferments vom Eiweiß sehen.

\section{Kapitel.}

Jetzt werden wir die Einwände betrachten, die zu verschiedenen Zeiten gegen die Identität der milchkoagulierenden und proteolytischen Fermente erhoben worden sind. Einer der stärksten Einwände wurde von Schmidt-Nielsen durch folgenden Versuch erhoben: der Verfasser nahm eine Pepsinlösung und ließ einen Teil derselben in der Kälte stehen, während er einen anderen Teil in den Thermostaten stellte. Nach einigen Tagen wurden beide Portionen mit dezinormaler Natriumlösung neutralisiert. Hierauf stellte der Verfasser nach der Koagulation äquivalente Mischungen her. Diese Mischungen ließ der Verfasser, nachdem sie angesäuert worden waren, verdauen, wobei die Portion aus dem Thermostaten bedeutend mehr verdaute. Man erhielt also ein Auseinandergehen beider Wirkungen und der Verfasser erklärt dies dadurch, daß das Chymosin schneller zerstört wird als das Pepsin. Wir können unsererseits nur die Richtigkeit dieser Beobachtung bestätigen, können aber dem Verfasser nicht in der Erklärung dieses Falles beistimmen. In der Tat sahen wir bei einer anderen Alkalisation ein Zusammenfallen beider Funktionen und bei der Neutralisation mit Natronlauge erhielten wir ein Zusammenfallen in den Fällen, wenn wir der Milch Säure beigefügt hatten. Man wird uns natürlich entgegenhalten, daß in diesem Falle das saure Pepsin gewirkt habe. Wir aber nehmen an, daß die Ursache der Erscheinung nicht die Zerstörung des Chymosins ist, sondern der Einfluß der Lauge auf das Pepsin in verschiedenen Milieus. Tatsächlich ist die chemische $\mathrm{Zu}-$ 
sammensetzung der Pepsinlösung, die in der Kälte gestanden hat, eine ganz andere als die der Lösung, die im Thermostaten gestanden hat und in dem sich das Eiweiß in Pepton verwandelt hat. Das Pepsin aber ist außerordentlich empfindlich gegen freies Alkali. Es erscheint nun die natürliche Frage, ob nicht das Eiweiß das Pepsin vor Veränderung schützen kann. Darum fing man an, dem Saft, der im Thermostaten gestanden hat, eine große Eiweißmenge beizufügen, und neutralisierte ihn dann mit Natron. Es ergab sich, daß die Portionen mit beigefügtem Eiweiß bedeutend schneller koagulierten; so teilten wir den Saft, der drei Tage im Thermostaten gestanden hat, in zwei Teile, $z u$ dem einen Teil fügten wir Eiweiß hinzu, zu dem anderen nicht. Nach der Neutralisation gerann der erstere in $6^{\prime}$, der letztere in $90^{\prime}$. Genau ebenso verfuhren wir mit dem Magensaft, der nicht im Thermostaten gestanden hatte. Die Portion mit dem hinzugefügten Eiweiß gerann in $10^{\prime}$, die ohne Eiweiß in 19'. Wir müssen also zugeben, daß bei der Neutralisation mit Natron irgend eine Einwirkung des Alkalis auf das Pepsin stattfindet, wobei das Eiweiß bis zu einem gewissen Grade das Pepsin vor Veränderungen schützt. Wenn nun aber bei der Neutralisation mit Natron nur eine Veränderung des Ferments stattfindet, so bedeutet dies, daß es möglich ist, durch eine Bearbeitung die Tätigkeit des Ferments wieder herzustellen und ein Zusammenfallen der beiden Funktionen zu erhalten. Und wirklich, nachdem man der Milch eine genügende Menge $\mathrm{CaCl}_{2}$ beigefügt hatte, fingen die äquivalenten Mischungen an, gleichmäßig zu verdauen, während von keinem sauren Pepsin die Rede sein konnte. $\mathrm{Zu}$ dem Versuch wurde Magensaft genommen. Portion A wurde mit $\mathrm{NaOH}$ neutralisiert, Portion $\mathrm{B}$ mit $\mathrm{NaHCO}_{3}$. Durch vorherige Proben wurden die ihrer Koagulation nach äquivalenten Mischungen mit einer großen Aenge $\mathrm{CaCl}_{2}$ hergestellt, wozu B 10 mal verdünnt wurde. Dann nahm man zur Koagulation je 0,3 Saft und verschiedene Mengen von $5 \% \quad \mathrm{CaCl}_{2}$; ohne Hinzufügen von $\mathrm{CaCl}_{2}$ nahm man je 1,0 Saft; Milch wurde je 5,0 genommen; zur Verdauung nahm man 1,0 äquivalente Mischung $+4,0$ Säure. 
Tabelle VI.

\begin{tabular}{r|r|r|r|r|r}
\hline \hline Menge des $5 \%$ igen $\mathrm{CaCl}_{2}$ & 0,7 & 0,2 & 0,1 & 0 & Verdaut \\
\hline A & $35^{\prime \prime}$ & $120^{\prime \prime}$ & $330^{\prime \prime}$ & $43^{\prime}$ & $2,3 \mathrm{~mm}$ \\
$70^{\prime \prime}$ & $150^{\prime \prime}$ & $11^{1 / \mathrm{z}^{\prime}}$ & 2,5,
\end{tabular}

Also erhält man beim Hinzufügen großer Mengen $\mathrm{CaCl}_{2}$ äquivalente Mischungen, die in gleicher Weise verdauen und koagulieren; mit der Verminderung der Menge $\mathrm{CaCl}_{2}$ tritt ein Auseinandergehen ein, das bei gänzlichem Mangel von $\mathrm{CaCl}_{2}$ am stärksten ist.

Und in der Tat mußten wir, nachdem wir die äquivalenten Mischungen nach der Koagulation ohne $\mathrm{CaCl}_{2}$ hergestellt hatten, B ungefähr 4 mal verdünnen und würden infolgedessen ein vollständiges Auseinandergehen in der Verdauungskraft erzielt haben. Also findet beim Stehen im Thermostaten gleichzeitig mit der Verminderung des Pepsins eine Veränderung in der Eiweißmenge statt. Bei der Neutralisation mit Ätznatron geht teils eine Zerstörung des Ferments, teils eine Veränderung desselben vor sich, und beide Einflüsse kommen um so schärfer zum Ausdruck, je stärker das Eiweiß verändert wird. Es ist wichtig zu bemerken, daß durch das Stehen im Zimmer eine Veränderung in den Säften vor sich geht, die mit $\mathrm{NaOH}$ neutralisiert wurden. So verglichen wir den Saft A, der mit $\mathrm{NaOH}$ neutralisiert war, mit dem Saft $\mathrm{K}$, den man mit $\mathrm{NaHCO}_{3}$ neutralisiert hatte. Nachdem wir die äquivalenten Mischungen nach Koagulation ohne $\mathrm{CaCl}_{2}$ hergestellt hatten, stellten wir die Säfte nach Hinzufügung von Säure zur Verdauung, wobei A 2,8 $\mathrm{mm}$ verdaute und $\mathrm{K} 2,0 \mathrm{~mm}$. Am zweiten Tage stellten wir nochmals Mischungen her, wobei $A$ in $4^{\prime} 45^{\prime \prime}$ koagulierte und 2,3 mm verdaute, während $\mathrm{K}$ in $4^{\prime} 30^{\prime \prime}$ koagulierte und $2,2 \mathrm{~mm}$ verdaute. Wenn wir durch den neutralisierten Saft $\mathrm{CO}_{2}$ hindurchgehen ließen, konnten wir diesen Prozeß beschleunigen und auch vollkommen die Wirkung wiederherstellen, indem wir den Saft erst ansäuerten und dann dialysierten.

Der Magensaft wurde mit $\mathrm{NaOH}$ neutralisiert (A); durch 
eine Portion (B) ließ man $\mathrm{CO}_{2}$ zwei Stunden lang hindurchgehen und ließ sie zwei Stunden unter Umrühren im Thermostaten stehen. Bei der dritten Portion (K) wurde der Magensaft mit $\mathrm{NaHCO}_{3}$ neutralisiert. Zur Koagulation nahm man je 5,0 Milch. Zuerst stellte man Äquivalente ohne Hinzufügung von $\mathrm{CaCl}_{2}$ her, dann eine andere Reihe mit Hinzufügung von

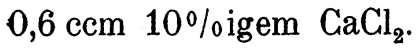

Tabelle VII.

\begin{tabular}{l|c|c|c|c|c|c|c}
\hline \multicolumn{1}{c|}{ Ohne $\mathrm{CaCl}_{2}$} & $\mathrm{~A}$ & $\mathrm{~B}$ & $\mathrm{~K}$ & $\mathrm{Mit} \mathrm{CaGl}_{2}$ & $\mathrm{~A}$ & $\mathrm{~B}$ & $\mathrm{~K}$ \\
\hline $1,0 \mathrm{ccm}$ Saft & $8^{\prime 1 / \mathbf{2}^{\prime}}$ & $3^{\prime} 50^{\prime \prime}$ & $\mathbf{4}^{\prime}$ & $0,6 \mathrm{GaGl}$ & $80^{\prime \prime}$ & $80^{\prime \prime}$ & $75^{\prime \prime}$ \\
$0,50 »+0,5$ Wasser & $\begin{array}{c}22^{\prime} \\
7^{\prime} 40^{\prime \prime}\end{array}$ & - & 0,1 Äquival. & - & - & - \\
$0,25 »+0,75$, & $\begin{array}{c}\text { in 1 St.nicht } \\
\text { geronnen }\end{array}$ & $15^{\prime} 30^{\prime \prime}$ & - & $\begin{array}{c}\text { Äquival. } \\
\text { verdauten }\end{array}$ & 2,7 & 2,8 & 2,9 \\
\hline Äquival. verdauten & 2,8 & 2,0 & 1,8 & &
\end{tabular}

Auf solche Weise müssen wir zugeben, daß das Auseinandergehen der Funktionen in Schmidt-Nielsens Versuche von dem Einfluß des Alkalis auf das Pepsin herrührt in Milieus mit verschiedenen Eiweißmengen, und nicht von der Zerstörung des Chymosins, da die koagulierende Wirkung (Labwirkung) wiederhergestellt werden kann. Folglich haben wir es mit einem besonderen Zustande des Pepsins $\mathrm{zu}$ tun, der infolge der Einwirkung des Alkalis entsteht. Zum Unterschied vom Fall des Dr. Tichomiroff wird hier das Pepsin durch Säure vollständig wiederhergestellt. Für diesen Zustand ist es charakteristisch, daß von $\mathrm{CaCl}_{2}$ eine Beschleunigung der Koagulation ausgeht, die einen größeren Umfang hat, als beim gewöhnlichen Pepsin (Tab. VI.). Dieses Verhalten dem $\mathrm{CaCl}_{2}$ gegenüber erinnert an Bangs $\left(^{1}\right)$ Beschreibung des Parachymosins. Die Ähnlichkeit geht noch weiter: in beiden Fällen verlangsamt sich die Zeit der Koagulation bedeutend mehr als der Grad der Verdünnung, wie dies aus der Tabelle VII ersichtlich ist.

Bei der Neutralisation mit $\mathrm{NaHCO}_{3}$ erhält man zuerst eine Vermehrung der Zeit proportionell der Verdünnung, die jedoch schnell ihre Grenze erreicht, worauf J. P. Pawlow 
und Parastschuk in ihrer Arbeit hingewiesen haben; hierauf verlangsamt sich die Koagulation. In Anbetracht dessen bezeichnet Bang ${ }^{\left({ }^{12}\right)}$ das milchkoagulierende Ferment im Magensaft des Hundes als Parachymosin. Dialysiert man jedoch den Magensaft des Hundes und macht ihn auf diese Weise neutral, so erhält man einfache umgekehrte Verhältnisse zwischen der Zeit der Koagulation und der Verdünnung. In dem unten angeführten Versuche wurde der Magensaft mit $\mathrm{NaHCO}_{3}$ neutralisiert; den anderen Saft dialysierte man; hierauf nahm man zur Koagulation je 1,0 mit Wasser verdünnten Saft auf 10,0 Milch.

\begin{tabular}{c|c|c}
\hline \multirow{2}{*}{$\begin{array}{c}\text { Relative } \\
\text { Fermentmenge }\end{array}$} & \multicolumn{2}{|c}{ Der Saft koagulierte } \\
& neutralisiert & dialysiert \\
\hline 1 & $2^{\prime}$ & $1^{\prime} 45^{\prime \prime}$ \\
$1 / 8$ & $4^{\prime}$ & $3^{\prime} 20^{\prime \prime}$ \\
$1 / 4$ & $7^{\prime} 50^{\prime \prime}$ & $6^{\prime} 10^{\prime \prime}$ \\
$1 / 8$ & $15^{\prime} 30^{\prime \prime}$ & $12^{\prime}$ \\
$1 / 18$ & $41^{\prime}$ & $25^{\prime}$ \\
$1 / 88$ & - & $51^{\prime}$
\end{tabular}

Auf solche Weise bemerkt man auch bei einer Neutralisation mit $\mathrm{HNaCO}_{3}$ eine leichte Veränderung des Ferments. Dies stimmt vollständig mit den Angaben Tichomirows ( $\left.{ }^{4}\right)$ überein, der gefunden hat, daß die Alkalisation mit Soda eine -Veränderung des Ferments herbeiführt, was aber sehr langsam vor sich geht. Also betrachteten wir das Parachymosin, als das erste Stadium der Alkaliwirkung auf das Pepsin. Es ist vollkommen verständlich, daß das Parachymosin der Alkaliwirkung gegenüber empfindlicher erscheint, als das Chymosin, d. h. das unveränderte Ferment. Dies ist nach Bang (11) der dritte charakteristische Zug für das Parachymosin.

Was nun die Behauptung Bangs anbetrifft, daß man durch die Erwärmung in neutraler Lösung das Pepsin zerstören könne und dadurch das Parachymosin ausscheiden, so können wir uns damit durchaus nicht einverstanden erklären. Wenn wir die mit $\mathrm{NaOH}$ neutralisierten Säfte, welche die für 
das Parachymosin charakteristischen Merkmale besaßen, oder auch neutrale Lösungen käuflicher Pepsinpräparate bis zu $76-77^{\circ}$ erwärmten, beobachteten wir immer eine deutliche proteolytische Wirkung.

Wir konnten jedoch wahrnehmen, daß die koagulierende Fähigkeit solcher erwärmter Säfte schärfer ausgesprochen war, als die Verdauungsfähigkeit. Gleichzeitig richteten wir unsere Aufmerksamkeit darauf, daß die Reaktion der Säfte nach der Erwärmung etwas alkalisch wurde. Außerdem findet ein reichliches Ausscheiden des geronnenen Eiweißes in dem erwärmten Saft statt. Die biuretische Reaktion ist im Filtrat sehr schwach. Man erhält eine äußerst schwache Alkalität und oft verursacht ein Tropfen Säure schon eine saure Reaktion. Darum fügten wir 1-2 Tropfen Säure hinzu und stellten die Mischung in den Dialysator, um eine neutrale Reaktion zu erhalten. Hierauf erhöhte sich die Verdauungsfähigkeit auffallend (von 0,9 auf 2,2 Serumstäbchen).

Von anderen Argumenten, die gegen die von uns verteidigte Theorie vorgebracht wurden, finden wir noch die Behauptung Glaessners, $\left({ }^{13}\right)$ daß im menschlichen Pankreassaft überhaupt keine Chymosinwirkung vorhanden sei. Diese Behauptung hat eine um so größere Bedeutung, da siê nur in Ausnahmefällen geprüft werden kann. Glücklicherweise ist kürzlich die interessante Arbeit Wohlgemuths über den Pankreassaft des Menschen erschienen. Dieser Autor hat bemerkt, was Glaessner übersehen hat. Wir führen hier die Schlußfolgerungen Wohlg e muths an, daß (S. 355) «. . . im menschlichen Pankreassaft sich ein Labferment findet, im inaktiven als Proferment, im aktiven als Ferment. Als zweites Faktum ergibt sich, daß die Aktivierung des Labferments mit der des Trypsins parallel geht, sei es, daß man mit Darmsaft oder mit Salzsäure den menschlichen Pankreassaft aktiviert». Wir werden hier nicht auf die Verfahren eingehen, die zur Trennung von Chymosin und Pepsin vorgeschlagen wurden, da dieselben in der Arbeit Pawlows und Parastschuks einer Kritik unterworfen worden sind. Wir können unsererseits nur hinzufügen, daß wir saure Aufgüsse aus Kälbermagen (Labmagen) her- 
stellten und sie in den Thermostaten stellten. Nicht ein einziges Mal konnten wir uns nach der Neutralisation mit $\mathrm{NaHCO}_{3}$ von der Abwesenheit der koagulierenden Wirkung überzeugen. Es ist allerdings wahr, daß bei langem Stehen die Koagulation sehr verzögert wurde; man brauchte nur zu der Milch $\mathrm{CaCl}_{2}$ (auf 5,0 Milch 1,0 ccm 5\% iger Lösung) in größerer Menge hinzuzufügen und die Koagulation fand in einigen Minuten statt; ohne Hinzufügung von Ferment aber stand die Milch mit $\mathrm{CaCl}_{2}$ stundenlang, ohne zu gerinnen. Wir müssen also zugeben, daß die koagulierende Fähigkeit überall und immer mit der proteolytischen Hand in Hand geht. Auf diese Weise existiert vorläufig noch nichts, was zugunsten des Vorhandenseins zweier Fermentgruppen spräche. Es scheint uns, daß sich die Annahme zweier solcher Fermentgruppen nur auf die Tradition gründet. Anders lassen sich einige Dinge gar nicht erklären. So konnte z. B. Hemmeter( $\left(^{5}\right)$ bei der Untersuchung des starken proteolytischen Ferments im Safte der Suava und der Ananas kein Chymosin entdecken, obgleich er einen hierher gehörigen Versuch anführt: «Obschon beide Säfte Milch koagulieren (ein Tropfen Ananassaft bringt 20,0 ccm Kuhmilch zur Gerinnung), konnten wir uns nicht überzeugen, daß diese Wirkung von einem Ferment abhing.» Wie groß müßte also der Säuregehalt dieser Fruchtsäfte sein, um die Fällung des Caseins in einer Verdünnung von 1 auf 200 herbeizuführen, und wäre es bei einem solchen Säuregehalt möglich, die reifen Früchte zu essen?

Die Annahme von 2 Fermentgruppen führt noch zu einer weiteren Komplikation. Sobald man eine Abweichung von den gewöhnlichen Regeln beobachtet, wird die Existenz eines neuen Ferments angenommen, obgleich die Abweichung von einer Veränderung der Reaktionsbedingungen ausgehen kann.

Wir halten es für notwendig, nochmals hervorzuheben, daß die Wirkungsregeln der Fermente in Verbindung mit der Zeit und Konzentration sich den Reaktionsbedingungen gemäß verändern und daß sich unter gewissen Bedingungen die Wirkungszeit der proteolytischen Fermente in einfachem Verhältnis zur Konzentration befindet. 
Dagegen häufen sich die Tatsachen, die zugunsten der Identität milchkoagulierender und proteolytischer Fermente spreçenen, mehr und mehr an. In der Arbeit Fleischers(15) wurde auf den Zusammenhang zwischen der gelatinespaltenden und milchkoagulierenden Wirkung der Bakterienfermente hingewiesen. Die Bakterienkulturen wurden mittels Filtration durch Chamberlandsche Kerzen sterilisiert; das Filtrat wurde neutralisiert und auf die Koagulation sterilisierter Milch hin, welcher man Säure beigefügt hatte, untersucht. Als Kontrolle dienten Portionen mit gekochtem Filtrat und Wasser. Es ergab sich, daß die Bakterienfiltrate, welche Gelatine flüssig machten, die Milch koagulierten, während diejenigen, unter deren Wirkung Gelatine nicht flüssig wurde, auch die Milch nicht gerinnen machten. Die Filtrate von b. acidi lactici koagulierten nicht, da die Koagulation der Milch mittels dieser Bakterien von der Bildung einer Säure, nicht aber eines Fermentes abhängt. Ferner hat der Autor gezeigt, daß das Anwachsen beider Funktionen bei Kulturen verschiedenen Alters parallel läuft. Endlich wirkten die Bakterienfiltrate nicht nur auf die Gelatine, sondern verdauten auch Eiweiß.

Kürzlich konnte nun Jacoby $\left({ }^{16}\right)$ ein proteolytisches Ferment in Labpräparaten nacbweisen, sowie eine parallele Verminderung beider Funktionen beim Erwärmen. Bei der Untersuchung der Wirkung der Antikörper auf das Chymosin fand der Verfasser «keinen Grund, welcher eine Spezifität der Antikörper gegen Lab und Pepsin sicherstellen und von dieser Seite aus eine Trennung notwendig machen könnte».

Die Annahme einer Identität der milchkoagulierenden und proteolytischen Fermente hat schon zu einer Erklärung des Einflusses der Alkalien auf das Pepsin geführt (Tichomirow); dieser Einfluß ist nämlich, als ein Übergang des Pepsins in einen latenten, inaktiven Zustand erklärt worden. Nur von der Annahme einer Identität ausgehend, konnten wir eine wirkliche Verdauungskraft des pylorischen Saftes entdecken. Im allgemeinen halten wir es für sehr wichtig, die eine Wirkung durch die andere zu kontrollieren. Dies schützt am besten gegen falsche Schlußfolgerungen. Die Proben auf Koagulation 
nehmen nicht viel Zeit in Anspruch, wenn man zu der Milch $\mathrm{CaCl}_{2}$ im Überfluß hinzufügt. Es wäre sehr wünschenswert, die Koagulationsmethode als klinisches Verfahren zwecks Bestimmung der Fermente zu prüfen. Um die Resultate vergleichen zu können, muß man den Mageninhalt mit Soda neu-

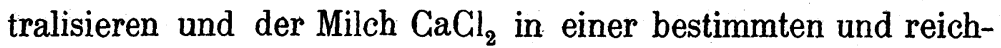
lichen Menge beifügen.

Als unsere Arbeit schon beendigt war, erfuhren wir mit Vergnügen, daß in Pekelharings Laboratorium Gewin(17) zu denselben Resultaten gekommen ist. Dieser Autor hielt das Parachymosin und das Chymosin für ein und dasselbe Ferment; der Unterschied zwischen ihnen wird durch die Eigenheiten der Beimischungen bedingt. Das Resultat des SchmidtNielsenschen Versuchs erklärt er durch die Zerstörung von Stoffen, die das Ferment schützen.

Zum Schluß spreche ich hochverehrtem Prof. Pawlow meinen Dank aus dafür, daß er mir das Thema vorgeschlagen hat, und für die Ratschläge, die er mir beim Ausführen dieser Arbeit erteilte.

\section{Literatur.}

1. Pawlow u. Parastschuk, ther die ein und demselben Eiweißferment zukommende proteolytische und milchkoagulierende Wirkung verschiedener Verdauungssäfte. Diese Zeitschrift, Bd. XLII.

2. Sawjalow, Zur Frage nach der Identität von Pepsin und Chymosin. Diese Zeitschrift, Bd. XLVI.

3. Ganike, Ein neues Verfahren zur Untersuchung der Fermentwirkung und über die doppelseitige Wirkung des Stärkeferments im Pankreassaft. Gaseta Botkinskaja 1901 (russisch).

4. Tichomirow, Zur Frage über die Wirkung der Alkalien auf das Eiweißferment des Magensaftes. Nachrichten der militärmedizinischen Akademie 1906 (russisch).

5. Hemmeter, Are the proteolytic and milkkoagulating effets of gastric and pancreatic juce due to one and the same enzyme? (Werden die proteolytischen und milchkoagulierenden Wirkungen des Magen- und Pankreassaftes durch ein und dasselbe Enzym verursacht?). Berl. Klin. Woch., 1905, Festnummer.

6. Nierenstein und Schiff, Über die Pepsinbestimmung nach Mett und die Notwendigkeit ihrer Modifikation für klinische Zwecke. Boos Ar., Bd. VIII. 
106 Sawitsch, Über milchkoagulierende und proteolytische Fermente.

7. Ganike, Über die physiologischen Bedingungen der Zerstörung und Konservierung der Fermente im Pankreassaft. Gaseta Bort. 1901 (russisch).

8. Vernon, The precipitality of pancreatic ferments by alcohol. (Die Fällungsfähigkeit der pankreatischen Fermente durch Alkohol.) Journ. of Physiol., Bd. XXIX.

9. Schrumpf, Darstellung des Pepsinferments aus Magenpreßsaft. Beiträge zur chem. Physiol. und Path., Bd. VI.

10. Schmidt-Nielsen, Über die vermeintliche Identität von Pepsin und Chymosin. Diese Zeitschrift, Bd. XLVIII.

11. Bang, Über Parachymosin, ein neues Labferment. Pflügers Arch., Bd. LXXIX.

12. Bang, Sind proteolytische und milchkoagulierende Fermentwirkung verschiedene Eigenschaften eines und desselben Ferments? Diese Zeitschrift, Bd. XLIII.

13. Glaessner, Über menschliches Pankreassekret. Diese Zeitschrift, Bd. XL.

14. W ohlg e muth, Untersuchungen über das Pankreas des Menschen. Über das Labferment. Bioch. Zeitschrift, Bd. II.

15. Fleischer, Zur Frage über die Wechselbeziehungen zwischen der proteolytischen und milchkoagulierenden Wirkung der von Bakterien herrührenden Fermente. Nachrichten der militärmedizinischen Akad. 1905 (russisch).

16. Jacoby, Über die Beziehungen der Verdauungswirkung und der Labwirkung. Biochem. Zeitschrift, Bd. I.

17. Koninkyke Akademie van Wetenschappen te Amsterdam. Proceedings of the Meeting 1907, S. 283. *An investigation of Mr. Gewin on the relation of pentin to chymosin $>$ by Pr. Pekelharing. 